\title{
Mobile Cross Reality for Cultural Heritage
}

\author{
Chris Davies \\ School of Computer Science \\ University of St Andrews \\ cjd44@st-andrews.ac.uk
}

\author{
Alan Miller \\ School of Computer Science \\ University of St Andrews \\ alan.miller@st-andrews.ac.uk
}

\author{
Colin Allison \\ School of Computer Science \\ University of St Andrews \\ ca@st-andrews.ac.uk
}

\begin{abstract}
Widespread adoption of smartphones and tablets has enabled people to multiplex their physical reality, where they engage in face-to-face social interaction, with Web-based social networks and apps, whilst emerging 3D Web technologies hold promise for networks of parallel 3D virtual environments to emerge. Although current technologies allow this multiplexing of physical reality and 2D Web, in a situation called PolySocial Reality, the same cannot yet be achieved with 3D content. Cross Reality was proposed to address this issue; however so far it has focused on the use of fixed links between physical and virtual environments in closed lab settings, limiting investigation of the explorative and social aspects. This paper presents an architecture and implementation that addresses these shortcomings using a tablet computer and the Pangolin virtual world viewer to provide a mobile interface to a corresponding 3D virtual environment. Motivation for this project stemmed from a desire to enable students to interact with existing virtual reconstructions of cultural heritage sites in tandem with exploration of the corresponding real locations, avoiding the adverse temporal separation caused otherwise by interacting with the virtual content only within the classroom. The accuracy of GPS tracking emerged as a constraint on this style of interaction.
\end{abstract}

\section{INTRODUCTION}

The rapid adoption of smartphones and tablets and their popularity for social interaction via the mobile Web [1] has led to people increasingly mixing their on-line and 'real life' behaviours, multiplexing traditional face-to-face social interaction with Web-based social networks and apps. The pervasive provision of these devices provides a new mechanism for people to take physical space for granted, to cerebrally occupy a Web-based location whilst their bodies are simultaneously established in a physical location [2]. The term PolySocial Reality (PoSR) has been proposed to describe these multiplexed mixed realities [3], wherein individuals interact within multiple environments [4], and to identify the extent and impact of shared and unshared experience in such situations [5]. Whilst current technologies allow PoSR involving 2D Web content to manifest, attempting the same with $3 \mathrm{D}$ content is marred by the "vacancy problem": the inability to immerse oneself in 3D content whilst maintaining awareness of one"s physical surroundings [6], the inability to simultaneously experience a sense of presence in both real and virtual environments.

The capacity of 3D virtual world environments to provide extensible collaborative platforms for the reconstruction of cultural heritage sites and the potential of such reconstructions to promote understanding of and engagement with cultural heritage content both in public and classroom settings has been explored in [7], [8]. The virtual worlds are programmable environments enabling the creation of historic scenes complete with sound, characters weather and rich in world interactions.
The 3D environments also act as an interface to wider educational resources in the form of text, images, sound and video. The historic scenes give a sense of place and stimulate the imagination whilst the linked materials provide accessible investigative pathways. Multiple control methodologies (traditional keyboard and mouse, XBox controllers and gesture recognition via Kinect) and display options (Oculus Rift, regular 24" desktop monitors, larger 40" televisions and 300 six megapixel projection) support heterogeneous deployment scenarios. These provide multiple modes of access to the same content; a network of reconstructions accessible via the Internet as part of the OpenSim hyper-grid; portable LAN exhibitions where multiple computers are connected to a server via local network suitable for classroom use; and immersive installations combining projection and Kinect for use in museums and cultural heritage centres.

In all these scenarios a recurrent theme has been the relationship between the virtual reconstruction and the physicality of the corresponding physical site. Frequently projects have involved interactions with the reconstruction and subsequent visits and tours of the physical site; however the temporal separation between these activities makes it harder to appreciate the sometimes complex relationships between the two. To overcome this temporal separation of experiencing the virtual and the real it is necessary for the virtual representation to be accessible in tandem at the physical site and for the vacancy problem to be addressed.

The cross reality concept [6], [9] addresses the vacancy problem and describes the mixed reality situation that arises from the combination of physical reality with a complete [10] 3D virtual environment. Previous cross reality experiments focused on static locations at which the two environments were linked within closed lab surroundings [2]. Cross reality concerns systems in which the virtual content constitutes a complete environment, as opposed to the sparse and discrete objects that augmented reality (AR) positions over a view of the real environment. This allows the virtual environment of cross reality systems to be accessed in absence of the real environment, promising interactions between on-site and remote virtual visitors, and more encompassing graphical content.

The contribution of this work is to extend virtual world functionality by developing and evaluating a mobile cross reality interface which supports interaction with 3D reconstructions of cultural heritage sites and simultaneous exploration of the corresponding physical site, thus providing a sense of presence in both the real site and the reconstruction. This is achieved using a tablet computer with location and orientation sensors and the Pangolin virtual world viewer [11]. 


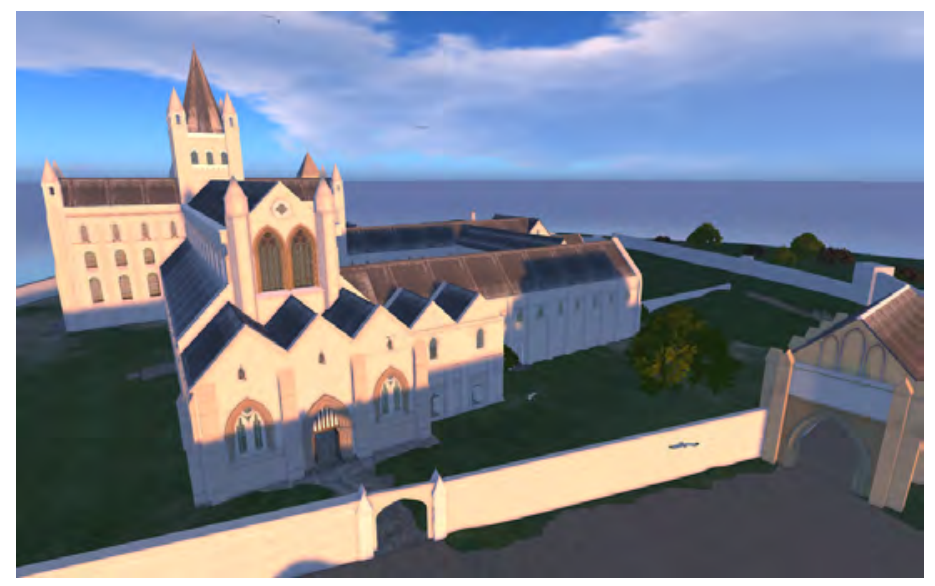

Fig. 1. Reconstruction of the St Andrews cathedral.

\section{SCOPE}

The extent that the real and virtual environments which constitute a cross reality system spatially correspond to each other is an important factor that limits the style of interactions possible. If the two environments have a high degree of spatial equivalence, that is to say that even if their visual appearances differ substantially that their fundamental layout and dimensions are the same such that navigating freely in one will not result in a collisions with objects in the other (an allusion to the 'mirror world' concept [12], [13], [14]), then monitoring a user's movements within the real environment provides a method for controlling their avatar within the virtual environment without the need for concious manual control. This approach substantially lightens the cognitive load of maintaining a presence in a virtual environment, which contributes to overcoming the vacancy problem.

This scenarios discussed here have a high degree of spatial equivalence between the real and virtual environments, as they deal with bringing together virtual reconstructions of cultural heritage sites with their corresponding real locations. The backdrop for many of the experiments is the impressive ruins of the St Andrews cathedral, while the virtual environment is a "distorted" [10] OpenSim simulation of the same location that presents a historically accurate reconstruction of the cathedral as it would have stood at the peak of its former glory $[8]^{1}$ (see figure 1). This is a large reconstruction, over $400 \mathrm{~m}$ by $600 \mathrm{~m}$, of a complex multi-storey building and thus represents a challenge for a mobile device to render and consequently is a challenging platform for testing.

Collaborations between computer scientists, educationalists and historians led to the creation of the St Andrews cathedral reconstruction [15], [16] and have also led to the creation of reconstructions of; a 6th Century Spartan Basilica [17], [18], Virtual Harlem (1921), Linlithgow Palace (1561), Brora Salt Pans (1599) [19], [20], Fethaston Fishing Station (19th century), Eyemouth Fort (1610), an Iron Age Wheel House and Caen Township (1815) [21]. These reconstructions provide a platform for interactive historical narratives, a stage for visitors to play upon and engage in both serious (and not so

\footnotetext{
${ }^{1}$ The reconstructions can be explored by setting up an account at www.openvirtualworlds.org/start and downloading a firestorm client
}

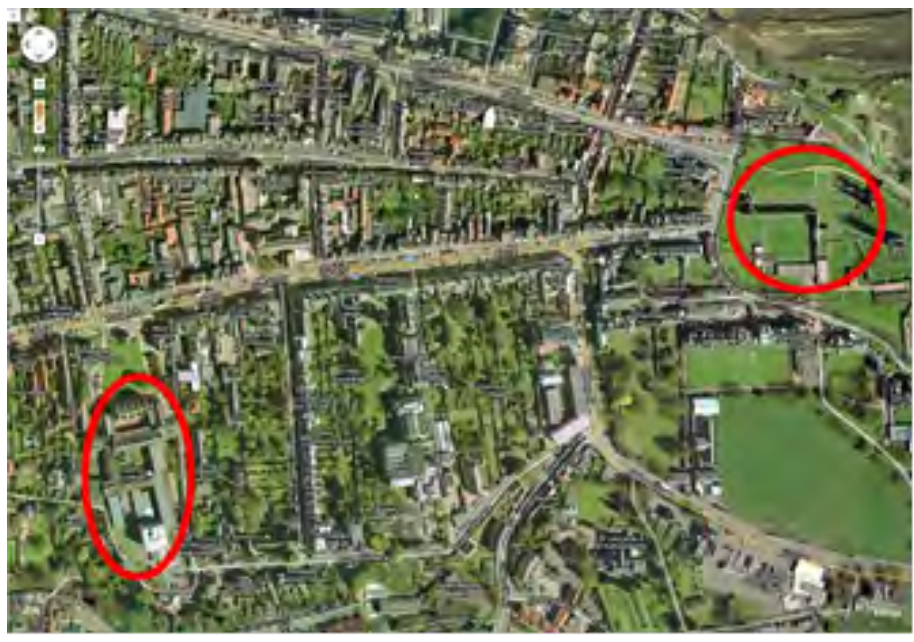

Fig. 2. Aerial photograph of St Andrews demonstrating the distance between Madras College (left ring) and the cathedral itself (right ring). The distance between the two sites is roughly $650 \mathrm{~m}$.

serious) games both alone and with other users, and serve as a focal point for educational investigations into local history and culture [7], [22]. The reconstructions have been widely used in a range of real world educational contexts. In the formal sector they have been a vehicle for investigative research, part of degree accredited university modules and used in both primary and secondary education. They have also been used as the content for interactive museum installations, art installations and community groups. This has involved further collaborations with Education Scotland, Historic Scotland, SCAPE Trust, Timespan Museum, the Museum of the University of St Andrews (MUSA), Madras College, Linlithgow Palace and Strathkiness Primary School.

Through extending the functionality of a virtual world client to support mobile exploration, the functionality associated with existing content is extended. Specifically mobile exploration is added to virtual world projects discussed above. It is also made available to other virtual archaeology hosted in OpenSim and SecondLife [27]. There has been considerable work in AR and VR which focus on the development of bespoke cultural heritage installations and is orthogonal to the work discussed in this paper [23], [24], [25], [26].

The project described in this paper develops a mobile interface to allow students to explore both a physical site and its virtual reconstruction in tandem, as well as being able to explore the reconstruction from a computer in the classroom and trying or from home. Figure 2 shows the spatial separation between the classroom and the physical site was during a session with students at St Andrews' Madras College. The project, introduced in [22], developed a modified version of the Second Life viewer called Pangolin, which through use of sensors allows movement of the avatar and camera to be implicitly controlled by sensing the physical position and orientation of the tablet computer which the user carries and upon which the viewer executes. Figure 3 depicts the system in use at the St Andrews cathedral.

This system promises to augment exploration of cultural heritage sites by allowing convenient navigation of the $3 \mathrm{D}$ reconstruction and stimulating reflection through the close 


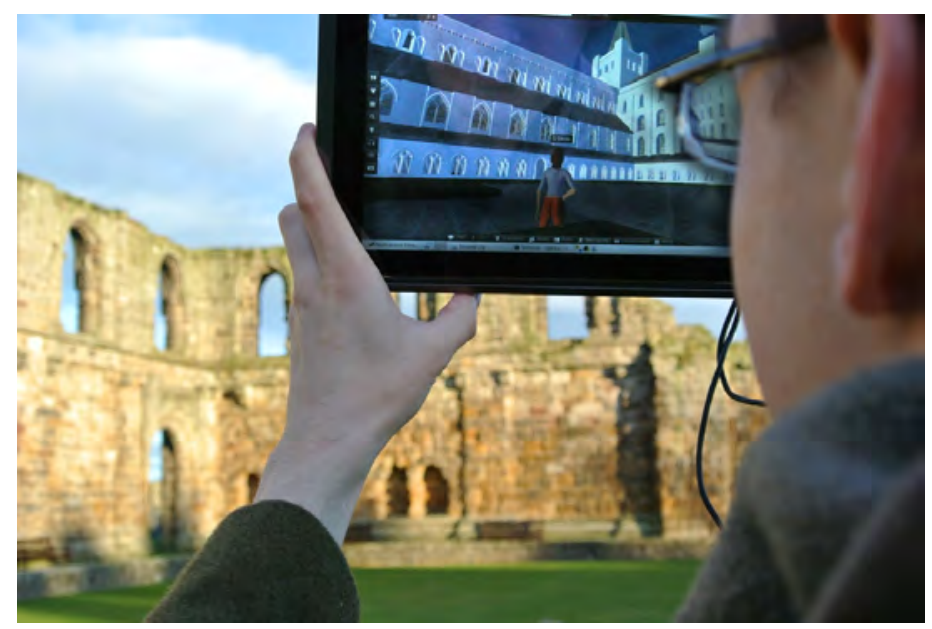

Fig. 3. The Pangolin viewer running on a tablet computer at the St Andrews cathedral, with the camera orientation of the viewer synchronised to the physical orientation of the tablet, the view of the virtual reconstruction corresponding to that of the physical ruins.

juxtaposition of the remains and an accessible interpretation. The use of a complete virtual environment also allows for the possibility of interaction between individuals and groups at the site with remote participants, including domain experts, who are connected to the reconstruction from a distant physical location.

\section{METHODS}

\section{A. Virtual Environment}

The 3D virtual environment component of the Pangolin system was implemented using the Second Life/OpenSimulator (SL/OpenSim) platform, which provides a 3D socialoriented multi-user non-competitive virtual environment which focuses on the community, creation and commerce [28] aspects of many users interacting within a shared space through the abstraction of avatars, rather than the competitive natures of games and the solitary environments commonly afforded by simulation and visualization platforms. The distributed client/server model of SL/OpenSim, wherein 3D content is stored on a grid of servers operated by a multitude of organizations and distributed to and navigated between by dispersed clients on demand when they enter a particular region rather than being pre-distributed as is the norm for games, simulations and visualizations, is analogous to the manner in which $2 \mathrm{D}$ social Web content is served from Web servers to client browsers and apps. This style of content delivery is necessary when considering the dynamic and ephemeral nature of consumergenerated media which constitutes the majority of the current 2D social Web and will make up the majority of expanding 3D social Web content.

Whilst SL/OpenSim encapsulates many of the desirable architectural features for 3D PoSR experiments it does not support execution upon familiar mobile platforms (Android/iOS) nor does it provision for avatar control from sensor data. However the open source nature of the SL viewer allowed modification to be effected, enabling control of the avatar and camera from real time data collected from position and orientation sensors connected to a tablet computer. This ability to

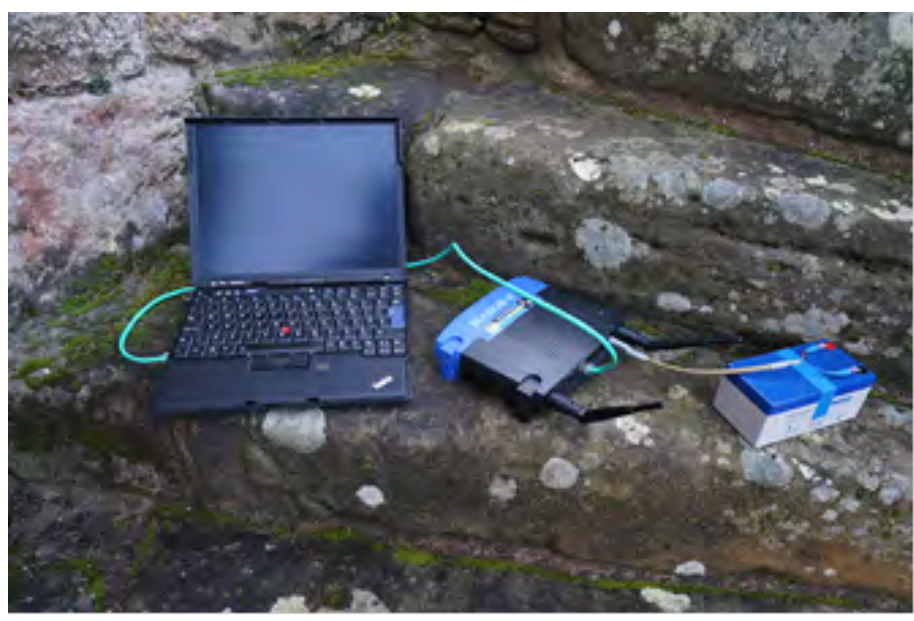

Fig. 4. Lenovo ThinkPad X61s laptop, Linksys WRT54G wireless router and sealed lead-acid battery providing OpenSim server via wireless to the $110 \mathrm{~W}$.

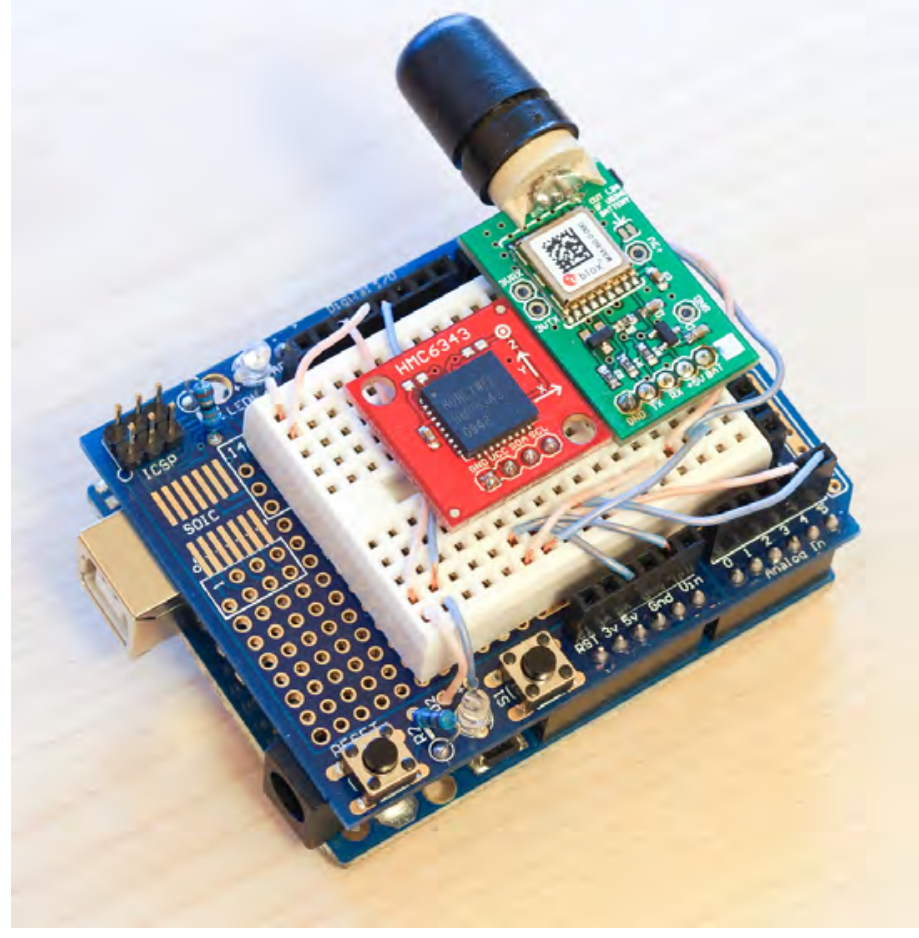

Fig. 5. The HMC6343 (red), MAX-6 (green) and SL-1202 (black protrusion) connected via a breadboard prototyping shield to the Arduino, in the setup and configuration that was then attached to the rear of the $110 \mathrm{~W}$ for the experiments.

control navigation within the 3D virtual environment without explicit conscious input of keyboard/mouse/touch commands is integral to reducing the cognitive load required to maintain a presence within a virtual environment which is a key requirement for overcoming the vacancy problem and achieving successful mobile cross reality.

As the SL viewer is only available for $\mathrm{x} 86$ platforms the choice of user hardware platform for the experiments was limited, with the MSI WindPad 110W presenting the most promising solution: a 10" tablet computer sporting an AMD Brazos Z01 APU (combining a dual-core x86 CPU and 


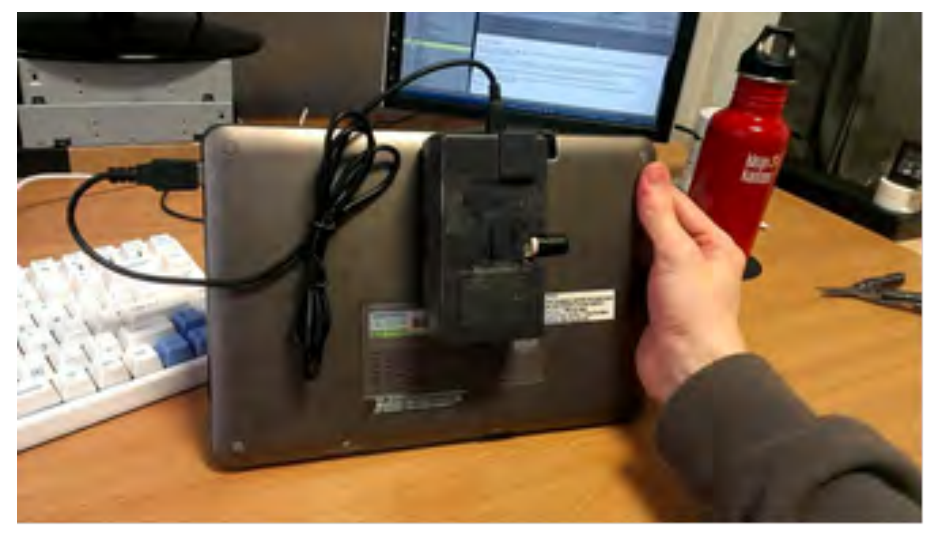

Fig. 6. The setup from gure 5 attached to the rear of the $110 \mathrm{~W}$. The sensors are congured such that (in the orientation of this photograph) the $\mathrm{X}$ axis is positive pointing straight down, $\mathrm{Y}$ is positive pointing straight right and $\mathrm{Z}$ is positive pointing perpendicular out of the rear face of the tablet.

Radeon HD6250 GPU). The user's position was monitored using GPS, a solution which is well suited to applications of the system within the use case of cultural heritage; such sites often constitute outdoor ruins at which a clear view of the sky allows for good GPS connectivity. For use cases where a similar modality of interaction is desired whilst indoors then an indoor positioning system would be used; a round up of such technologies is available in [29].

Due to there being no computer infrastructure installed at the site and to reduce computational load on the $110 \mathrm{~W}$, the OpenSim server was run on a separate Lenovo ThinkPad X61s laptop computer during the experiments. Due to the limited range of the laptops wireless interface, the laptop was connected by RJ45 Ethernet cable to a Linksys WRT54G wireless router to allow the $110 \mathrm{~W}$ to access the OpenSim server wirelessly from anywhere within the experiment area. The router was powered from a $12 \mathrm{~V}$ sealed lead-acid battery. This set-up is shown in figure 4.

\section{B. GPS Configuration}

The 110W features an AzureWave GPS-M16 GPS receiver; however limited API provision lead to use of a separate u-blox MAX-6 GPS receiver outfitted with a Sarantel SL-1202 passive antenna. The MAX6 is of higher operational specification than the GPS-M16 and supports Satellite Based Augmentation Systems (SBAS) which improve the accuracy of location data by applying additional correction data received from networks of satellites and ground-based transmitters separate to those of the GPS system. These networks include the European Geostationary Navigation Overlay Service (EGNOS) that covers the UK where the experiments took place.

The product summary for the MAX-6 claims accuracy of 2.5m Circular Error Probable (CEP) without SBAS corrections and $2 \mathrm{~m}$ CEP with SBAS corrections demonstrated with a good active antenna. This means that, in an ideal situation with SBAS correction data available, there would be $50 \%$ probability that each position reported by the GPS receiver would be within $2 \mathrm{~m}$ of its actual position. The SL1202 antenna used is passive, however as the distance between antenna and the MAX-6 IC itself in the hardware application is only a few millimetres there would have been negligible benefit from using an active antenna.

The MAX-6 was operated in "pedestrian" dynamic platform model, use of SBAS correction data was enabled and frequency of readings was set to the maximum of $5 \mathrm{~Hz}$.

To determine the real world accuracy attainable with the MAX-6 outfitted with the SL-1202 in situations akin to those of the cultural heritage case study, a walking route around the St Andrews cathedral ruins, akin to the route that an individual visitor or school group might take, was planned and then walked with the MAX-6 connected to a laptop computer via an Arduino operating as a Universal Asynchronous Receiver/Transmitter (UART) feeding the raw National Marine Electronics Association (NMEA) messages into the 'ucenter' GPS evaluation software version 7.0 which logged the messages for later evaluation. Simultaneously for comparative purposes a mid-range consumer Android smartphone was used to record the same track; a HTC One $S$ containing a gps One Gen 8A solution within its Qualcomm Snapdragon S4 processor and using Google's "My Tracks" app version 2.0.3 to record the data. The three sets of positional data (planned route, MAX-6 recorded route and smartphone recorded route) were entered into a PostgreSQL database [28, 29] and the PostGIS database extender"s ST HausdorffDistance algorithm was used to calculate the Hausdorff distances between the recorded routes and the planned route and between the recorded routes themselves. In this scenario, the Hausdorff distance represents the furthest distance needed to travel from any point on the route recorded by the GPS receiver to reach the nearest point on the planned route. Because of the substantially greater inaccuracies identified in the latter part of the recorded tracks, separate Hausdorff distances were calculated both for the complete tracks and also for truncated first and second subtracks.

Translating real world positions, obtained via the GPS receiver as latitude and longitude pairs, into corresponding OpenSim $(\mathrm{X}, \mathrm{Y})$ region coordinates is achieved using the haversine formula [30] from spherical trigonometry. The prerequisites for this approach are that the OpenSim model is aligned correctly to the OpenSim compass as the real location is aligned to real bearings (although provision to specify an offset within the Pangolin viewer for non-aligned models would be a trivial addition), that the model was created to a known and consistent scale and that a single anchor point is known for which both the real world latitude/longitude and corresponding OpenSim $(\mathrm{X}, \mathrm{Y})$ region coordinates are known.

Using the haversine formula the great-circle (or orthodromic) distance between the latitude of the anchor point and the latitude of the new GPS reading is calculated, then applying the scale of the model results in the equivalent distance in OpenSim metrics between the $\mathrm{Y}$ coordinate of the anchor point and the $\mathrm{Y}$ coordinate of the position corresponding to the new GPS reading. Repeating the same calculations with the longitude of the new GPS reading provides the distance between the $\mathrm{X}$ coordinate of the anchor point and the $\mathrm{X}$ coordinate of the position corresponding to the new GPS reading. Adding or subtracting these distances as appropriate to the OpenSim coordinates of the anchor point provides the OpenSim coordinates that correspond to the new GPS reading, to which the avatar is then instructed to move. 


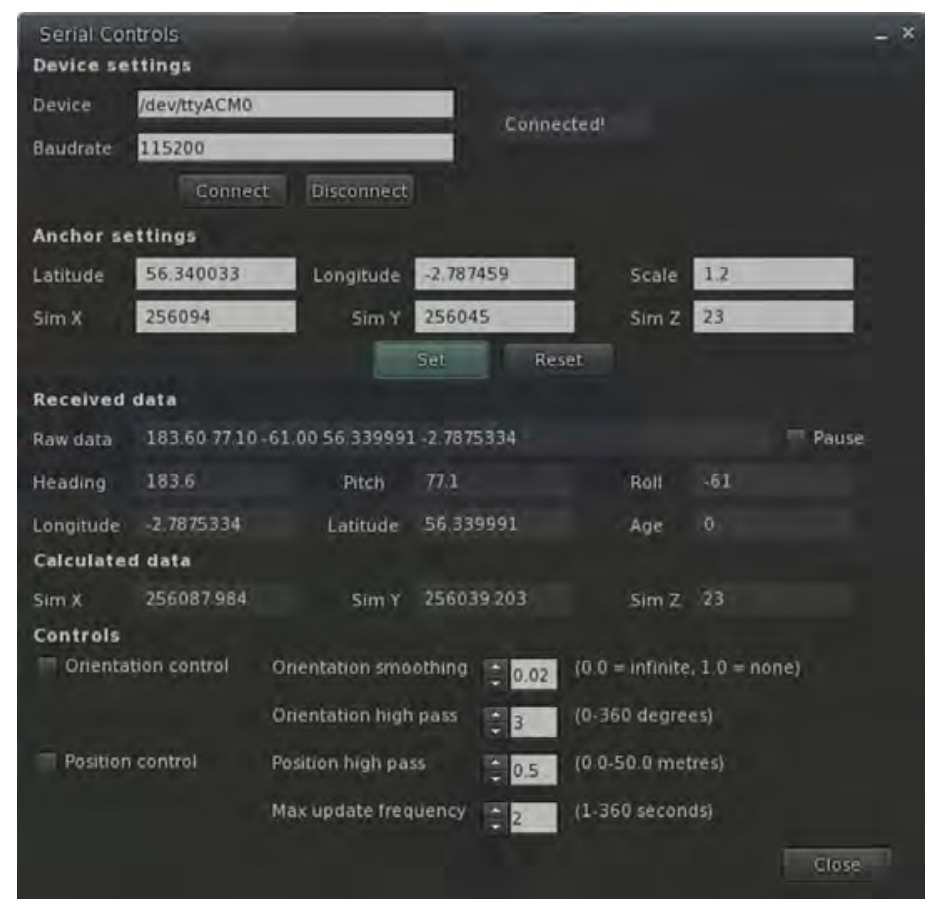

Fig. 7. The GUI within the Pangolin viewer that allows administration of the position and orientation control of the avatar. In this screenshot Pangolin is connected to the Arduino and is receiving position and orientation data..

The anchor point is specified using global coordinates, not local coordinates. This allows navigation to operate across region boundaries and within mega regions (it is not limited to a single $256 \times 256$ meter OpenSim region) and there are no restrictions for the placement of the OpenSim component of the anchor point (it can be anywhere in any region, movement of the avatar can be in any direction from it (positive and negative), it does not have to be at the centre of the model or even in a region that the model occupies). Calculating a global coordinate is simply a case of multiplying the position of the region by 256 and then adding the local coordinate. For example, for an anchor at local coordinate (127, 203, 23) within a region that is at $(1020,1042)$ the global $\mathrm{X}$ coordinate is calculated as $\left(\begin{array}{ll}1020 & 256\end{array}\right)+127=261247$ and the global $\mathrm{Y}$ coordinate as $(1043256)+203=267211$. Elevation $(\mathrm{Z})$ is ignored due to a combination of the relatively low accuracy of these data attainable via GPS (when compared to the longitudinal/latitudinal accuracy) and as the case study explored involved users navigating outdoor ruins remaining at ground level.

\section{Orientation}

To control the SL camera in the required fashion, sensor data is collected for the direction that the user is facing (in terms of magnetic compass bearing) and the vertical angle (pitch) at which they are holding the tablet. Magnetic compass bearing is sensed using a magnetometer and pitch by an accelerometer. Roll data is also captured by the accelerometer, however it was expected that users would keep the tablet in a roughly horizontal fashion when interacting with it, thus using these data to control the SL camera's roll was not deemed to be beneficial and was not implemented.
The 110W does not feature a magnetometer and its tilt sensor is rudimentary (only useful for differentiating between discrete cases of landscape and portrait orientation for screen rotation). Several alternative sensors were auditioned, including the MMA8452, ADXL335, HMC5883L and eventually the HMC6343 which was adopted for the experiments. The HMC6343 combines a 3-axis magnetometer, 3-axis accelerometer and algorithms to internally apply the accelerometer"s readings to tilt compensate the magnetometer"s readings; tilt compensation is necessary for an accurate compass bearing when the device is not held in a perfectly level orientation, such as when the user tilts it up or down to view content above or below their eye level.

Magnetic declination information was entered into the HMC6343 for the position of the cathedral and the date of our experiments. The HMC6343s hard-iron offset calculation feature was used each time the hardware configuration was altered. The sampling frequency of the HMC6343 was set to its highest value of $10 \mathrm{~Hz}$. Orientation was set to upright front to match the physical orientation of the IC in the experiments (see gure 6).

\section{Interfacing GPS/Orientation hardware with $S L$}

The MAX-6 and HMC6343 were connected to an Arduino (the setup used throughout the experiments is shown in gure 5) and a sketch (the name given to programs that execute upon the Arduino platform) written to receive the data from the ICs, perform simple processing upon them and relay them to the tablet via USB connection [32]. The TinyGPS library was used to abstract processing of NMEA messages from the MAX-6 to obtain the required latitude and longitude values.

Leveraging standard SL avatar/camera control interfaces was explored by programming the Arduino to mimic a standard USB HID joystick via the Lightweight USB Framework for AVRs (LUFA), sending messages that the viewer interpreted as coming from a joystick and allowing the use of the standard joystick options. However the granularity of control attainable via this method was not sufficient and thus the viewer was modified (giving rise to the Pangolin viewer) to make use of the Boost.Asio $\mathrm{C}++$ library to support receiving data via serial port and to use these data to control the movement of the avatar and camera by directly interfacing with the control functions at a lower level of abstraction. Receipt of messages is performed in an asynchronous non-blocking fashion, with the viewer"s main loop processing the most recently received message in each iteration. Messages follow the format

\section{(bearing)(pitch)(roll)(latitude)(longitude)}

The viewer"s GUI was modified with the addition of a dialogue that allows the user to specify the path of the serial device, separately enable or disable sensor-driven camera and movement control, as well as providing numerous controls for fine-tuning its behaviour, including the ability to specify highpass filters for avatar movement and specify the smoothing applied to camera control. This GUI also presents the necessary fields for input of the anchor point details and fields for diagnostic output of the received information. Figure 7 shows this GUI within the Pangolin viewer. 


\section{RESUlts}

Two plausible modalities of interaction were identified for this system, with each presenting different requirements with regards to accuracy of position tracking.

The first modality is one in which a number of locations that represent points of particular interest are identified. This is already a common practice at cultural heritage sites, with such locations often bearing signs or placards presenting text and/or images explaining what can be observed from the position. With Pangolin, when a user walks within a certain range of such a point, their avatar can be moved to the corresponding location within the reconstruction (and a sound played to alert the user to the fact that there is something of interest to observe) from which they can then move the tablet around them to examine their surroundings in the reconstruction. This modality is similar to audio tours employed by many museums and cultural heritage sites, but replaces the requirement to follow a static route or type in numbers of locations with the ability to freely navigate the real environment with access to additional information being triggered automatically once within the required range of a point of interest.

The second modality is one of free roaming exploration, in which the movements of the user's avatar within the reconstruction mimic the user's movements within the real world as closely as possible. The first modality can be scaled to function with different accuracies of position tracking; as long as the distance between any two points of interest is at least as much as the worst case performance of the position tracking then distinguishing correctly between different points will always succeed. The second modality requires extremely accurate position tracking, arguably surpassing the capabilities of mainstream GPS technology even in ideal situations.

During the experiments the MAX-6 was unable to maintain reception of the additional correction data required for SBAS operation; when left stationary for several minutes reception was possible however subsequent movement of only a few meters at walking pace broke the connection. This reduced the theoretical maximum performance of the unit to $2.5 \mathrm{~m}$ CEP, with observed performance being lower. Figure 8 depicts an aerial view of the St Andrews cathedral ruins; the blue line represents the planned route, red the route recorded by the MAX-6 receiver and green the route recorded by the smartphone for comparative purposes, both while walking the planned route.

The Hausdorff distance between the planned route and that recorded by the MAX-6 was $1.02 \mathrm{e}^{04}$. The 'length' of a degree of latitude and a degree of longitude depends upon location upon the Earth; around the location of the St Andrews cathedral $1^{0}$ of latitude is equivalent to $111347.95 \mathrm{~m}$ and $1^{0}$ of longitude to $61843.88 \mathrm{~m}$. Thus the Hausdorff distance of $1.02 \mathrm{e}^{04}$ can be visualized as $11.3 \mathrm{~m}$ of North/South inaccuracy or $6.3 \mathrm{~m}$ of East/West inaccuracy (or a combination of both $\mathrm{N} / \mathrm{S}$ and E/W inaccuracy not exceeding a total displacement of $1.02 \mathrm{e}^{04}$ from the planned route). The MAX-6 did achieve better performance than the smartphone, which recorded a Hausdorff distance of $1.33 \mathrm{e}^{04}(14.8 \mathrm{~m} \mathrm{~N} / \mathrm{S}, 8.2 \mathrm{~m} \mathrm{E} / \mathrm{W})$. The Hausdorff distance between the routes logged by the MAX6 and the smartphone was $1.14 \mathrm{e}^{04}(12.7 \mathrm{~m} \mathrm{~N} / \mathrm{S}, 7.0 \mathrm{~m} \mathrm{E} / \mathrm{W})$, which represents a low correlation between the inaccuracies recorded by the two receivers even though they are of similar magnitudes from the planned route.

The maximum inaccuracies were recorded when walking along the South wall of the cathedral's nave. This wall is one of the most complete sections of the building with stonework reaching some $30 \mathrm{ft}$ above ground level and providing an effective obstruction to line-of-sight to half of the sky (and substantially impairing reception of signals from GPS satellites) when in close proximity to it. When considering just the sub-route shown in figure 9, which terminates before this wall begins to significantly obstruct view of the sky, the Hausdorff distances are notably smaller; the MAX-6 achieved a Hausdorff distance of $7.23 \mathrm{e}^{05}(8.05 \mathrm{~m} \mathrm{~N} / \mathrm{S}, 4.47 \mathrm{~m} \mathrm{E} / \mathrm{W})$ throughout this sub-route, with the smartphone still behind with $8.99 \mathrm{e}^{05}(10.01 \mathrm{~m} \mathrm{~N} / \mathrm{S}, 5.56 \mathrm{~m} \mathrm{E} / \mathrm{W})$. Again the Hausforff distance between the receivers showed low correlation between the inaccuracies, at $6.43 \mathrm{e}^{05}(7.12 \mathrm{~m} \mathrm{~N} / \mathrm{S}, 3.98 \mathrm{~m} \mathrm{E} / \mathrm{W})$.

When analysing the tracks in the vicinity of the nave (see gure 10) it is shown that although the MAX-6 outperformed the smartphone in terms of Hausdorff distance this relationship can be considered misleading as the smartphone track corresponded more closely in shape to the planned route even if it did stray further at its extreme. The discrepancy in the behaviour of the two receivers in this situation is attributed to different implementations of dead-reckoning functionality between the receivers. Dead-reckoning is the process used when a GPS receiver loses reception of location data from satellites and extrapolates its position based upon a combination of the last received position data and the velocity of travel at the time of receiving these data.

Pangolin's camera control from orientation data does not have as stringent performance criteria as the movement control from position data. Unlike augmented reality where sparse virtual content is superimposed upon a view of a real environment and the virtual objects must be placed accurately in order for the effect to work well, cross reality presents a complete virtual environment that is viewed separately or side-by-side with the real environment and thus discrepancies between orientation of real and virtual environments have a less detrimental effect to the experience.

Although the accuracy of the camera control during the experiments was reported as being sufficient, the speed at which the camera orientation moved to match physical orientation was reported as being too slow, resulting in having to wait for the display to 'catch up' to changes in orientation. This is attributed to the $10 \mathrm{~Hz}$ sampling rate of the orientation sensors which, particularly after readings are combined for smoothing purposes to reduce jerky movement, resulted in too infrequent orientation updates. Frame rates within Pangolin whilst navigating the route averaged between 15 and 20 frames per second with the viewer"s quality and speed slider set to the low position. The style of explorative interaction with virtual content that this system employs is more resilient to input lag and low frame rates than other scenarios of interaction with virtual content such as fast paced competitive video games including First Person Shooters (FPS) [31], but overall user experience would nonetheless be improved by a faster sampling of orientation data and a higher frame rate. Additionally it should be noted that the cathedral reconstruction was created with relatively powerful desktop computers in 


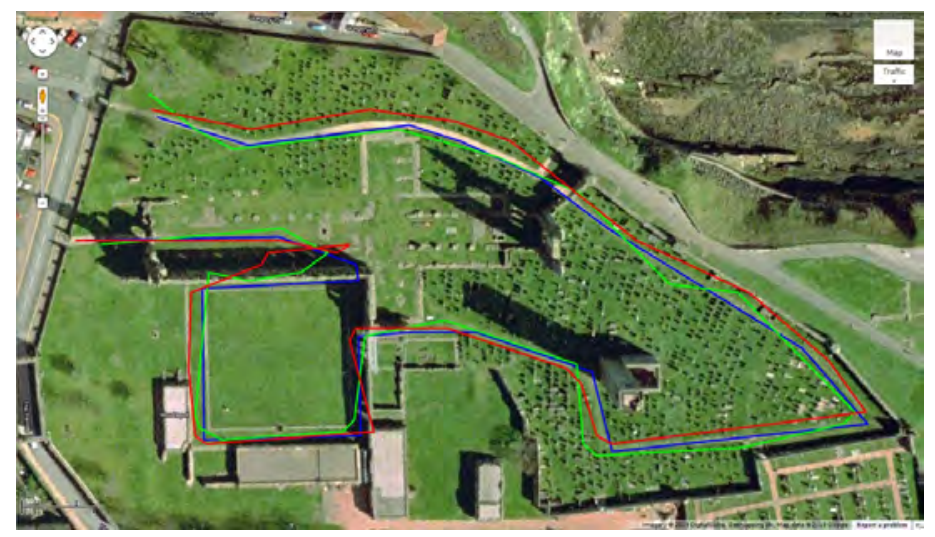

Fig. 8. Aerial view oriented North upward of the St Andrews cathedral ruins; the blue line represents the planned route, red the route recorded by the MAX-6 and green the route recorded by the smartphone whilst walking the planned route.

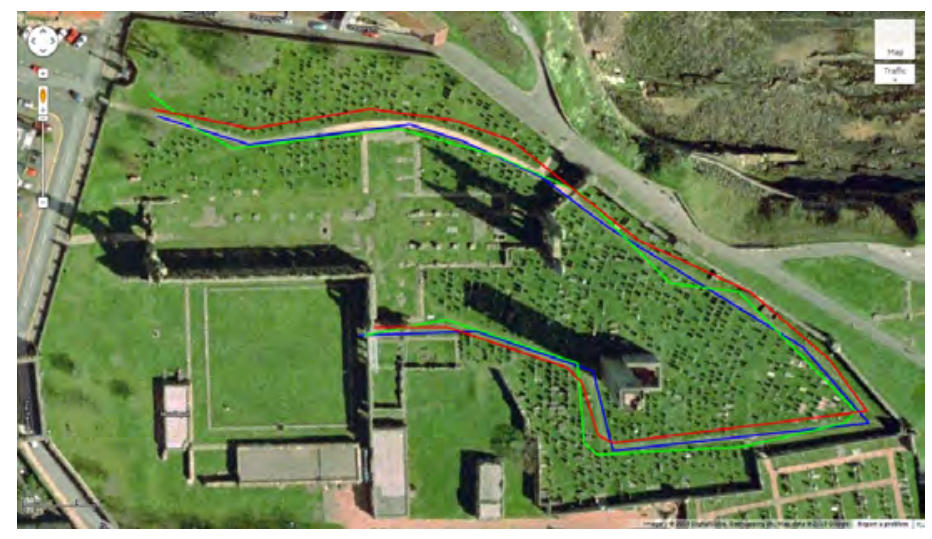

Fig. 9. Aerial view oriented North upward of the St Andrews cathedra ruins; the blue line represents the first sub-route of the planned route, red the sub-route recorded by the MAX- 6 and green the sub-route recorded by the smartphone whilst walking the first planned sub-route.

mind as the primary deployment platform and has not been optimized for use on less powerful mobile platforms such as Pangolin. Performance of Pangolin on a less graphically complex OpenSim region (Salt Pan 2 [19]), that also depicts a reconstruction of a cultural heritage site, was better at 20 to 25 frames per second at the low position and between 15 and 20 frames per second at high (see figure 11).

\section{INTERPRETATIONS}

The results demonstrate that the system enables a user to walk around a physical heritage site and to use a tablet to view the reconstruction of the site. The user can simultaneously commentary, in the form of text, audio, video and images, relevant to their specific location within the site. The accuracy of GPS limits the accuracy of synchronisation between the physical and virtual views.

The positional accuracy of $1.02 \mathrm{e}^{04}$ attained by the MAX-6 is sufficient for the first modality of interaction (that of distinguishing and navigating between multiple points of interest). This value of $1.02 \mathrm{e}^{04}$ (analogous to a combination of $11.3 \mathrm{~m}$ of North/South inaccuracy or $6.3 \mathrm{~m}$ of East/West inaccuracy) represents a constraint on the granularity of the content; it is the

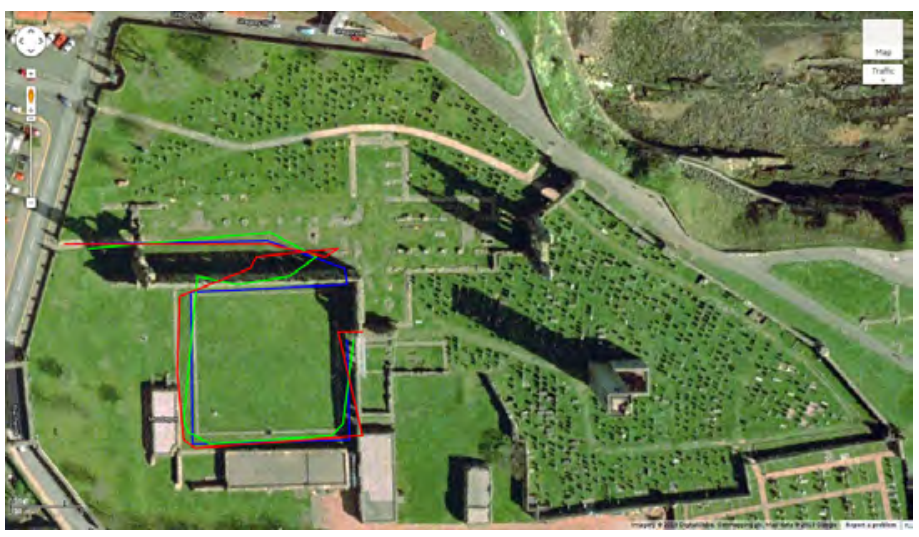

Fig. 10. Aerial view oriented North upward of the St Andrews cathedral ruins; the blue line represents the second sub-route of the planned route, red the sub-route recorded by the MAX- 6 and green the sub-route recorded by the smartphone whilst walking the second planned sub-route.

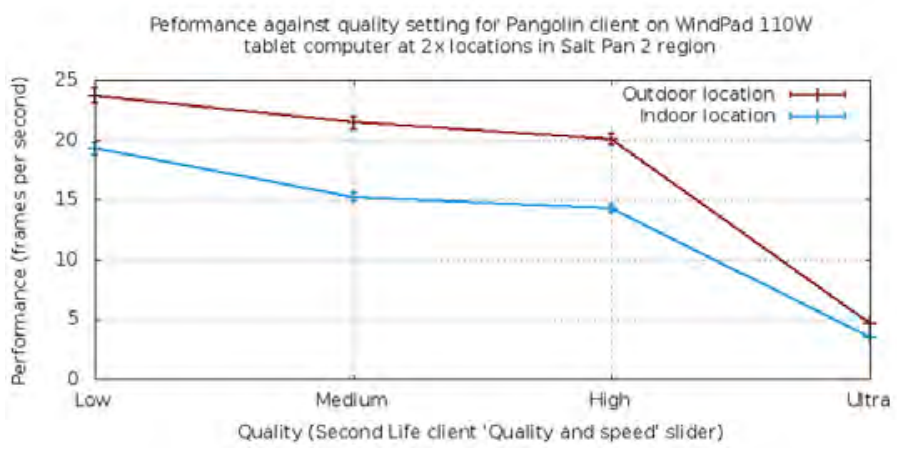

Fig. 11. Plot of Pangolins performance (measured in frames per second) against different graphical settings (selected via the Quality and speed slider of the viewer) in two positions within the Salt Pan 2 region.

minimum distance required between any two points of interest for them to be correctly differentiated between. This same value is not sufficient for the second modality of interaction (that of free roaming exploration with avatars mimicking their users movements as closely as possible). This modality would require the use of additional position tracking techniques to improve accuracy to around $1 \mathrm{~m}$ CEP (analogous to $8.98 \mathrm{e}^{06}$ latitude or $1.62 \mathrm{e}^{05}$ longitude around the location of the St Andrews cathedral).

Use of a GPS receiver that is lower performance than the MAX-6 used by Pangolin, but more common due to being of the calibre integrated into smartphones and tablets such as that used in the experiments, is still sufficient for the rst modality but with a larger minimum distance required between any two points of interest. The Hausdorff distance of $1.33 \mathrm{e}^{04}$ recorded by the smartphone used in the experiments is analogous to $14.8 \mathrm{~m} \mathrm{~N} / \mathrm{S}$ or $8.2 \mathrm{~m} \mathrm{E} / \mathrm{W}$ around the location of the cathedral.

Observed accuracy of the orientation tracking is sufficient for both modalities of interaction; the accuracy of orientation tracking required does not change with different positional accuracy and the accuracy of orientation attained in the experiments is sufficient for an acceptable user experience, however the experience would benefit from better graphical quality and higher responsiveness to changes in user orientation. As tablets increase in capability user experience will improve. 


\section{CONCLUSIONS}

Manifestations of PoSR for 2D content are commonplace, yet the ability to forge PoSR situations involving 3D content remains elusive. As 3D Web technologies develop, the demand for 3D PoSR will grow. The cross reality concept, when freed from static linking between physical and virtual environments, provides a technique to address this shortcoming. The Pangolin virtual world viewer provides a mobile, location and orientation aware cross reality interface to spatially related 3D virtual environments. Pangolin extends the use of 3D virtual world environments. It enables students to learn from reconstructions of cultural heritage content, by allowing them to interact with such reconstructions whilst simultaneously exploring the corresponding physical environments.

Performance of position tracking by GPS emerged as a constraint upon the modality of interaction possible using commercially available non-assisted GPS receivers. Those of the quality built into smartphones and tablets proved capable of supporting the points of interest modality but not the free roaming exploration modality.

These conclusions hold for today's commodity technology. We can expect the resolution, processing power and rendering capability of mobile phones and tablets to continue to increase for any fixed price point. Similarly, augmented positioning systems providing greater positional accuracy are likely to emerge. Thus we conclude that the benefits of having accurate virtual interpretations of historic locations available at the sites in a mobile fashion will be available for school visits, cultural heritage investigation and tourists of the future. As mobile 3D cross reality technology becomes common place and matures, applications in education, entertainment, business and the arts will emerge that will surprise us all.

\section{REFERENCES}

[1] Accenture. (2012, September) Mobile web watch Internet usage survey. WWW. Accenture. [Online]. Available: http://www.accenture.com/SiteCollectionDocuments/PDF/AccentureMobile-Web-Watch-Internet-Usage-Survey-2012.pdf

[2] S. A. Applin and M. D. Fischer. (2011, Feb.) A cultural perspective on mixed, dual and blended reality. WWW. Centre for Social Anthropology and Computing, University of Kent Canterbury. IUI Workshop on Location Awareness for Mixed and Dual Reality. [Online]. Available: http://archive.org/details/anthropunk

[3] _ - "Pervasive computing in time and space: The cultural context of "place" integration," in Proceedings of the Seventh International Conference on ?Intelligent Environments, 2011, pp. 285-293.

[4] _ - "Polysocial reality: Prospects for extending user capabilities beyond mixed, dual and blended reality. , dfki, (2012), 14." in LAMDa12, 2012, pp. 1-4.

[5] _ (2012) Visualising poly social reality. Just in TIme Sociology. [Online]. Available: http://jitso.org/2012/12/03/visualising-polysocialreality-revised/

[6] J. Lifton, "Dual reality an emerging medium," Ph.D. dissertation, Massachusetts Institute of Technology, Department of Media Arts and Science, 2007.

[7] C. Allison, A. C. Allison, C. J. Davies, L. Dow, S. Kennedy, A. Miller, I. Oliver, and I. Perera, "Growing the use of virtual worlds in education: an opensim perspective," in Proceedings of 2nd European Summit EiED, 2012.

[8] S. Kennedy, A. Miller, L. Dow, I. Oliver, R. Sweetmand, A. Campbell, J. McCaffery, C. Allison, D. Green, J. Luxford, and R. Fawcett, "Living history with open virtual worlds: Reconstructing st andrews cathedral as a stage for historic narrative," in Proceedings of 2nd European Immersive Education Summmit EiED, 2012.
[9] J. A. Paradiso and J. A. Landay, "Cross-reality environments," IEEE Pervasive Computing, vol. 8(3), pp. 14-15, July 2009.

[10] J. Lifton and James, "Dual reality: Merging the real and the virtual," in First International ICST Conference on Facets of Virtual Environments (FaVE), 2009.

[11] C. J. Davies. (2013) Pongolin source code.

[12] D. Gelenter, Mirror Worlds: or the Day Software Puts the Universe in a Shoebox....How it Will Happen and What it Will Mean. Oxford University Press (USA), 1993.

[13] A. Bar-Zeev. (2007, July) Second earth. Reality Prime. [Online]. Available: http://www.realityprime.com/blog/2007/07/second-earth/

[14] W. Roush. (2007) Second earth. MIT Technology Review. [Online]. Available: http://www.technologyreview.com/article/408074/secondearth/

[15] A. Miller, L. Dow, S. Kennedy, and R. Fawcett, "Exploring cultural heritage with open virtual worlds: the reconstruction of st andrews cathedral in opensimulator," in Proceedings of the International Congress on Digital Herritage, 2014.

[16] R. Fawcett, The architecture of the Scottish medieval Church. Yale University Press, 2011.

[17] K. Getchell, A. Miller, J. R. Nicoll, R. Sweetman, and C. Allison, "Games methodologies and immersive environments for virtual fieldwork." IEEE Transactions on Learning Technologies, vol. 3, pp. 281293, 2010.

[18] R. Sweetman and E. Katsara, "The acropolis basilica project, sparta: A preliminary report for the 2000 season," British School at Athens, Tech. Rep., 2002.

[19] T. Dawson, A. Vermehren, A. Miller, S. Kennedy, and I. Oliver, "Digitally enhanced community rescue archaeology," in Proceedings of first International Congress on Digital Heritage, 2013.

[20] J. Hambly, "Brora back beach, sutherland data structure report," SCAPE, Tech. Rep., 2010.

[21] J. McCaffery, A. Vermehren, K. Strikland, S. Kennedy, and C. Lefley, "Exploring heritage through time and space: supporting community reflection on the highland clearances," in Proceedings of first International Congress on Digital Heritage, 2013.

[22] C. J. Davies, A. Miller, and C. Allison, "Virtual time windows: Applying cross reality to cultural heritage," in Proceedings of PGNet 2012, 2012.

[23] D. Weng, D. Cheng, Y. Wang, and Y. Liu, "Display systems and registration methods for augmented reality applications." Optik - International Journal for Light and Electron Optics, vol. 123,9, pp. 769-774, 2012.

[24] A. Foni, G. Papagiannakis, and N. Magnenat-Thalmann, "A taxonomy of visualization strategies for cultural heritage applications." $A C M$ Journal on Computing and Cultural Heritage, vol. 3.1, pp. 1-21, 2010.

[25] J. Carmigniani, B. F. amd M. Anisetti, P. Ceravolo, E. Damiani, and M. Ivkovic, "Augmented reality technologies, systems and applications." Multimedia Tools and Applications., vol. 51(1), pp. 341-377, 2011.

[26] G. Papagiannakis, S. Schertenleib, M. Ponder, M. Arevalo, N. Magnenat-Thalmann, and D. Thalmann, "Real-time virtual humans in ar sites." in Proc. of IEE Visual Media Production (CVMP04), London, 204.

[27] L. M. Sequeira and L. C. Morgado, "Virtual archaeology in second life and opensimulator," Journal of Virtual Worlds Research, vol. 6.1, pp. 3-18, 2013.

[28] Y. Sivan, "3d $3 \mathrm{c}$ real virtual worlds defined: The immense potential of merging 3d. community, creation and commerce," Journal of Virtual Worlds Research, vol. 1(1), pp. 1-32, 2008.

[29] R. Mautz, "Indoor positioning technologies," Ph.D. dissertation, ETH Zurich, 2012.

[30] K. A. Hirsch and H. Reichardt, The VNR Concise Encyclopedia of Mathematics, W. Gellert, H. Kustner, M. Hellwich, and H. Katner, Eds. Van Nostrand Reinhold New York 2nd Edition, 1977.

[31] M. Claypool and K. Claypool, "Latency and player actions in online games," Commun. ACM, vol. 49, no. 11, pp. 40-45, 2006. 